Nervenarzt 2009 $\cdot 80: 1273-1274$ DOI 10.1007/s00115-009-2864-4

Online publiziert: 24 . Oktober 2009

(c) Springer Medizin Verlag 2009

F. Schneider ${ }^{1}$ W. Maier ${ }^{2}$

${ }^{1}$ Klinik für Psychiatrie und Psychotherapie, Universitätsklinikum Aachen

${ }^{2}$ Klinik und Poliklinik für Psychiatrie und Psychotherapie, Bonn

\title{
Psychiatrie in der Lebensspanne
}

von englischsprachigen Protagonisten besonders aus Europa dominiert.

der Kongress der Deutschen Gesellschaft für Psychiatrie, Psychotherapie und Nervenheilkunde (DGPPN) findet auch in diesem Jahr wieder in Berlin statt. Er wird sich in mehr als einer Sicht von den vorangegangenen Veranstaltungen unterscheiden. Zum einen hat die DGPPN es sich zur Aufgabe gemacht, interessierte Medizinstudenten für unser Fach zu interessieren und zu begeistern. Deshalb wurden inzwischen 750 Studentenstipendien vergeben, die sicherlich den einen oder anderen in seinem Interesse an unserem Fach bestärken und so hoffentlich in naher $\mathrm{Zu}$ kunft noch mehr geeigneten Nachwuchs für unser Fach zu gewinnen. Wir sind uns natürlich darüber im Klaren, dass dieses eine Maßnahme ist, die nicht isoliert betrachtet werden darf - der DGPPN-Vorstand hat einen Masterplan zum Thema Nachwuchs im Fach aufgestellt und stellt die Nachwuchsförderung in den Vordergrund seiner Arbeit. Dazu gehören z. B. auch zahlreiche neue wissenschaftliche Preise, ausgelobt insbesondere für den wissenschaftlichen Nachwuchs.

Ein anderer Punkt, der Ihnen sicher auffallen wird, ist die starke Zunahme englischsprachiger Veranstaltungen. Etwa $20 \%$ aller Veranstaltungen in diesem Jahr werden in einem englischen Track zusammengefasst und größtenteils simultan übersetzt. Die DGPPN möchte damit keineswegs die Bedeutung der Veranstaltung als größter deutschsprachiger Psychiatriekongress in Frage stellen, aber auf der anderen Seite ist es auch unser elementares Interesse, frühzeitig in neueste Entwicklungen in Klinik und Forschung involviert zu sein. Diese werden größtenteils auf Englisch kommuniziert und oft
Im höheren Lebensalter sind unbestreitbar demenzielle Erkrankungen von großer individueller und sozioökonomischer Bedeutung. Diesem Wandel über die Lebensspanne trägt die DGPPN nicht zuletzt mit einem speziellen Veranstaltungstag zum Deutschen Zentrum für Neurodegenerative Erkrankungen (DZNE) Rechnung. So wird am Freitag des Kongresses unter anderem Professor Dr. Nicotera, Gründungsdirektor des DZNE, einen Plenarvortrag halten.

Bei aller berechtigten Aufmerksamkeit für Demenzen darf aber bei Patienten im höheren Lebensalter nicht übersehen werden, dass auch Depressionen, Angsterkrankungen und Wahnsyndrome im Alter, Benzodiazepinabhängigkeit oder missbrauch sowie alterstypische Persönlichkeitsakzentuierungen und -störungen eine große klinische Relevanz haben.

\section{- In diesem Heft versuchen wir, an einzelnen Beispielen die Aufmerksamkeit auf eine ganzheitliche Betrachtung des Menschen über die Lebensspanne zu richten.}

Der Einfluss genetischer Faktoren auf Fähigkeiten und Verhalten wird meist als konstanter, lebenszeitübergreifender Faktor angesehen. Dem ist aber nicht so. Fritz Poustka und Wolfgang Maier stellen beispielhaft für kognitives Leistungsvermögen und Störungen die sich mit dem Alter wandelnden genetischen Einflussgrößen dar.

Kerstin Konrad und Michael Rössler stellen in ihrem Beitrag den Verlauf des Aufmerksamkeitsdefizit-/Hyperaktivitätssyndroms (ADHS) über die Lebens- 
spanne dar; obwohl ADHS oft als Störung des Kindes- und Jugendalters wahrgenommen wird, besteht es bei mehr als der Hälfte der Betroffenen bis ins Erwachsenenalter fort. Die am stärksten akzentuierten Auffälligkeiten weichen allerdings von denen im jüngeren Alter ab. Erst seit relativ kurzer Zeit wird ADHS in diesem Sinne als chronische psychische Erkrankung wahrgenommen. Doch nur wenn sich diese Einschätzung durchsetzt, also erst vor dem Hintergrund einer Betrachtung von ADHS über die gesamte Lebensspanne, werden sich die Fragen nach den genetischen und neurobiologischen Ursachen bzw. Korrelaten befriedigend beantworten lassen.

Irene Neuner und Andrea Ludolph stellen mit dem Beispiel des Tourette-Syndroms ein Krankheitsbild vor, welches mit ersten Tics bereits im frühen Kindes- und Jugendalter beginnt, sich bis ca. zum 11. Lebensjahr weitestgehend manifestiert und bis ins höhere Erwachsenenalter bestehen bleibt. Während im höheren Alter die Tics zum Teil milder werden, gibt es eine Reihe von Patienten mit einer fortwährenden erheblichen Krankheitsbelastung. Bei richtiger Einschätzung der Symptomatik und eventueller Komorbiditäten stehen mittlerweile eine ganze Reihe aussichtsreicher therapeutischer Optionen zur Verfügung, von der Verhaltenstherapie über die Pharmakotherapie bis in seltenen Fällen hin zur tiefen Hirnstimulation.

Viola Jucksch und Kollegen stellen die Persönlichkeitsentwicklung im Kindesund Jugendalter in den Vordergrund. Auffälligkeiten in der Persönlichkeit bei Kindern und Jugendlichen können nicht als Persönlichkeitsstörungen diagnostiziert werden, da zu diesem Zeitpunkt die Entwicklung der Persönlichkeit noch nicht als abgeschlossen gilt. In dieser Phase der Lebensspanne kann man am ehesten von Persönlichkeitsentwicklungsstörungen sprechen, die einigen Studien zufolge sogar höhere Prävalenzen als entsprechende Persönlichkeitsstörungen im Erwachsenenalter aufweisen.

Dieser Darstellung von Persönlichkeitsstörungen in den ersten Lebensphasen stellen Hans Förstl und Kollegen ihren Überblicksbeitrag „Persönlichkeit im höheren Lebensalter" gegenüber. Während in dem Bereich der Gerontopsychia- trie demenzielle Erkrankungen oder Depressionen im Zentrum der Aufmerksamkeit stehen, werden Persönlichkeitsakzentuierungen oder -störungen oft nicht hinreichend beachtet. Dargelegt werden hier insbesondere das Fortbestehen von definierten Persönlichkeitsstörungen, aber auch der Zusammenhang von Persönlichkeitsveränderungen und kognitiven Beeinträchtigungen bis hin zur Demenz.

Karl Mann und Mitarbeiter richten den Blick auf die Bedeutung von Suchterkrankungen über die Lebensspanne und beginnen folgerichtig mit der schädlichen Wirkung von Alkohol auf die Entwicklung des Babys im Mutterleib. Besondere Aufmerksamkeit ist aber im Zusammenhang mit Suchterkrankungen vor allem auf das Jugendalter zu richten, da hier nach aktueller Einschätzung die Weichen für den Umgang mit Suchtmitteln im weiteren Leben gestellt werden. Auf der anderen Seite scheint derzeit aber auch vor allem die Zahl der Suchterkrankungen im höheren Lebensalter zuzunehmen. Neben dem zunehmenden Alkoholkonsum stehen hier insbesondere Medikamentenabhängigkeiten im Fokus.

Die Schwerpunktbeiträge werden abgeschlossen durch einen Beitrag von Jürgen Fritze, der vor dem Hintergrund der aktuellen Gesundheitsgesetzgebung diskutiert, inwiefern man von einer Versorgungsgerechtigkeit über die Lebensspanne ausgehen kann. Oder plakativ gefragt, erlaubt es unser Gesundheitssystem, ältere Menschen mit psychischen Erkrankungen genauso intensiv und kostspielig zu behandeln wie Menschen im mittleren Lebensalter? Und: Wie verhält es sich diesbezüglich mit Kindern und Jugendlichen?

Wir hoffen, Sie teilen unser Interesse an der Betrachtung psychischer Störungen des Menschen über die gesamte Lebensspanne.

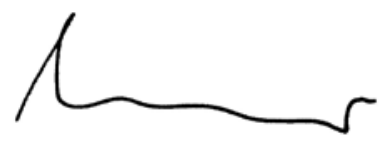

Prof. Dr. Frank Schneider, Aachen

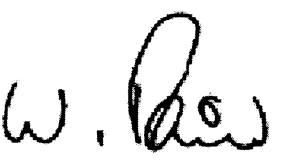

Prof. Dr. Wolfgang Maier, Bonn

\section{Korrespondenzadresse}

Prof. Dr. Dr. F. Schneider

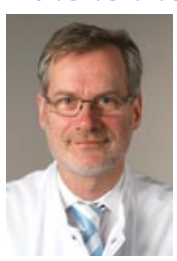

Klinik für Psychiatrie und Psychotherapie, Universitätsklinikum Aachen 52074

Pauwelsstraße 30Aachen fschneider@ukaachen.de 